Rabaska

Revue d'ethnologie de l'Amérique française

RABASKA

\title{
Alan Lomax en Louisiane : les hauts et les bas d'un chercheur de terrain
}

\section{Alan Lomax in Louisiana: Trials and Triumphs of a Fieldworker}

\section{Barry-Jean Ancelet}

Volume 6, 2008

URI : https://id.erudit.org/iderudit/019979ar

DOI : https://doi.org/10.7202/019979ar

Aller au sommaire du numéro

Éditeur(s)

Société québécoise d'ethnologie

ISSN

1703-7433 (imprimé)

1916-7350 (numérique)

Découvrir la revue

Citer cet article

Ancelet, B.-J. (2008). Alan Lomax en Louisiane : les hauts et les bas d'un chercheur de terrain. Rabaska, 6, 21-40. https://doi.org/10.7202/019979ar
Résumé de l'article

Cet article ne prétend pas repenser ici le travail considérable de ceux qui ont récemment étudié la vie et l'oeuvre de John et d'Alan Lomax sur les plans national et international. Il se limite plutôt à l'impact des Lomax en Louisiane française, surtout d'Alan, personnage qui se montrait à la fois inspirant et intimidant, frustrant et fascinant. Pendant les années 1930, John et Alan Lomax ont parcouru ce pays dans le but de réaliser des enregistrements de musique traditionnelle pour la Library of Congress. Leurs enregistrements sont devenus la base de l'Archive of Folk Song à la Library of Congress, véritable bijou de la musique traditionnelle des États-Unis. On dit d'Alan Lomax que ses observations étaient brillantes, tandis que ses interprétations passaient quelquefois à côté de la question. L'exposé ne cherche ni à glorifier ni à avilir, mais à reconnaître dûment le mérite et la critique dans la mesure où ils s'appliquent. 


\title{
Terrains
}

\section{Alan Lomax en Louisiane : les hauts et les bas d'un chercheur de terrain ${ }^{1}$}

\author{
BARRY-JEAN ANCELET \\ Centre d'études acadiennes et créoles \\ Université de Louisiane à Lafayette
}

Je devrais commencer en mettant les choses au clair. D'abord, je ne prétends pas repenser ici le travail considérable de Porterfield, $\mathrm{Hirsch}^{2}$ et d'autres qui ont récemment étudié la vie et l'œuvre de John Lomax et son fils Alan sur les plans national et international. Je limiterai plutôt mes remarques à l'impact des Lomax, surtout d'Alan, en Louisiane française, le milieu au sein duquel je suis engagé depuis le début des années 1970. Je devrais également souligner que ma propre expérience avec ce dernier s'est avérée plutôt inconstante. Alan Lomax se montrait à la fois inspirant et intimidant, frustrant et fascinant. Selon un collègue, ses observations étaient brillantes, tandis que ses interprétations passaient quelquefois à côté de la question. Je ne cherche ni à glorifier, ni à avilir, mais à reconnaître dûment le mérite et la critique dans la mesure où ils s'appliquent.

Pendant les années 1930, John et Alan Lomax ont parcouru ce pays dans le but de réaliser des enregistrements de musique traditionnelle pour la Library of Congress. Leur travail ressemblait à d'autres projets de l'époque de la Dépression comme le Farm Securities Administration's Photographer's Project et le Work Projects Administration's Federal Writer's Project qui avaient pour objectif de documenter les États-Unis d'Amérique. Ces projets représentaient une transformation de l'auto-représentation étatsunienne, à mesure que le creuset nationaliste du président Théodore Roosevelt laissait la place aux attitudes pluralistes qui soulignaient la richesse de la diversité ethnique, culturelle et linguistique. Les enregistrements Lomax sont devenus

1. Cet article s'inspire de la communication en l'honneur de Phillips Barry que j'ai présentée à l'assemblée annuelle de l'American Folklore Society en 2003 à Albuquerque, Nouveau-Mexique. Merci à Nicole Boudreau qui a préparé la traduction préliminaire de ce texte.

2. Jerrold Hirsch, Portrait of America : A Cultural History of the Federal Writers'Project, Chapel Hill, University of North Carolina Press, 2003 ; Nolan Porterfield, Last Cavalier : The Life and Times of John A. Lomax, 1867-1948, Champaign/Urbana, University of Illinois, 2001. 
la base de l'Archive of Folk Song à la Library of Congress, véritable bijou de la musique traditionnelle des États-Unis.

Les enregistrements sur le terrain destinés aux archives montraient que chaque tradition de chansons se jouait selon des modes distincts sur le plan culturel et que la meilleure façon de se documenter sur ces traditions était de les enregistrer sur place à l'aide d'appareils portatifs. De fait, l'invention de l'enregistreur portatif de disques, que nous avons pu apporter avec nous sur le terrain dans les années 1930, a changé le cours même de la musicologie et des études folkloriques. Il était désormais possible d'enregistrer des traditions musicales orales directement dans leur contexte naturel. Pour le chercheur sur le terrain, il était profondément satisfaisant d'observer la fierté et le plaisir des gens de la communauté quand ils entendaient pour la première fois l'enregistrement de leur propre musique et se rendaient compte que cet art était désormais préservé de façon permanente ${ }^{3}$.

Les enregistrements Lomax de chansons cadiennes et créoles en Louisiane témoignent de l'évolution des objectifs de John, qui s'orientait originalement vers les ballades anglo-américaines. Il a entrepris cette excursion en Louisiane française à l'aide de son fils précoce Alan - il n'avait alors que 18 ans - qui avait déjà étudié un peu le français. Alan a expliqué plus tard que John, qui se consacrait à l'époque à la rédaction de son livre Adventures of a Ballad Hunter, aurait donné carte blanche à son fils, muni de la machine enregistreuse de la Library of Congress. « Comme je parlais quelques mots de français et que je voulais me prouver ce dont j'étais capable et comme mon père était occupé à écrire son autobiographie (John Lomax, Adventures of a Ballad Hunter, New York, Macmillan, 1949), il s'est tourné vers moi pour assurer la majeure partie du travail sur le terrain ${ }^{4}$. »

Cependant, les enregistrements de 1934 montrent que John était effectivement présent à plusieurs des entretiens. Il a même dicté la mission de l'expédition : rendre les enregistrements accessibles aux musiciens des générations futures ( « [...] à la disposition des musiciens des générations à venir $[\ldots] »)$. Il semble aussi qu'Alan aurait réalisé lui-même certains des enregistrements. Par exemple, sa visite à la communauté créole noire de la région de Jennings / Lake Arthur a donné lieu aux premiers enregistrements de la tradition du juré, c'est-à-dire l'ensemble de rondes et de cris a cappella associé à la communauté créole noire française et anglaise, précurseur de ce

3. Alan Lomax, «Introduction», Louisiana Cajun and Creole Music, 1934: The Lomax Recordings, Swallow Records 8003-2, 1988 ; nouvelle édition augmentée sous le titre Alan and John Lomax : The Classic Louisiana Recordings, Cajun and Creole Music, 1934/1937, vol. I (Rounder 116611842-2) et vol. II (Rounder 11661-1843-2), 1999 [désormais Alan Lomax, LCCM].

4. Alan Lomax, LCCM. 
que l'on appelle aujourd'hui le zydeco . Il y a quelques indications intéressantes que la connaissance de la langue française du jeune Alan était mise en cause par les Cadiens et les Créoles qui parlaient des variétés nonstandard du français, et qui faisaient l'objet de ses enregistrements. Dans un extrait de son émission nationale Folksong Train à l'antenne de la radio Mutual Broadcasting dès 1948, Alan raconte l'essentiel de l'histoire correctement, mais il y a ces quelques détails gênants :

Nous allons désormais vers le sud et l'ouest, en traversant l'Alabama et le Mississippi pour nous rendre en Louisiane et en traversant la Louisiane jusqu'à la côte. Nous y découvrons une espèce de valse paresseuse, sauvage et féroce. Ils l'appellent « Jolie Blonde ». C'est la musique cadienne fais-do-do, et je vais vous dire de quoi il s'agit dans un instant. Vous savez, ils appellent cette musique « fais-do-do », ce qui veut dire « endors-toi ». Je ne sais pas comment ils s'imaginent qu'on arrive à s'endormir avec tout ce raffut... « Jolie Blonde ». Vous savez, on pourrait appeler cela de la musique française tropicale. L'histoire est la suivante : Évangéline et son peuple sont venus d'Arcadie au dix-septième siècle. Ils se sont installés dans ces terres marécageuses et ils ont pris eux-mêmes une tournure tropicale. Leur langue a changé, leur musique a changé et écoutez maintenant ce que cela a donné. Alors disons « au revoir » à la musique fais-dodo et quittons la Louisiane pour passer directement de l'autre côté du golfe, où...6

Je ne vois pas tout à fait comment une valse peut être à la fois paresseuse, sauvage et féroce. D'ailleurs, ses références historiques et géographiques sont erronées. Les ancêtres des Cadiens ne sont pas arrivés en Louisiane au $\mathrm{XVII}^{\mathrm{e}}$ siècle. Ils sont venus suite à leur exil d'Acadie (la Nouvelle-Écosse actuelle) en 1755. De plus, Évangéline est un personnage fictif créé par l'auteur anglo-américain Henry Wadsworth Longfellow vers le milieu du $\mathrm{XIX}^{\mathrm{e}}$ siècle. Il est intéressant de noter qu'il utilise comme musique de fond la nouvelle version de Jolie Blonde, enregistrée par Harry Choates, plutôt que la version de 1929 des Breaux Brothers, beaucoup moins commerciale et travaillée, ou encore l'un de ses propres enregistrements provenant de la Louisiane française en 1934, dont beaucoup sont bien plus « tropicaux » et « sauvages et féroces ». Il appelait déjà la musique cadienne « fais-do-do », confondant l'événement social et la musique qu'on y joue. Il a continué de

5. Barry-Jean Ancelet, «Zydeco / Zarico : The Term and the Tradition », dans James Dormon (dir.), The Creoles of the Gulf Coast, Knoxville, University of Tennessee Press, 1996 ; Michael Tisserand, The Kingdom of Zydeco, New-York, Arcade Publishing, 1998 ; Ben Sandmel and Rick Olivier, Zydeco ! Jackson, University Press of Mississippi, 1999.

6. Alan Lomax, Extrait de l'émission de radio nationale « Folksong Train», Mutual Broadcasting Company, 1948 (document sonore fourni par Nick Spitzer). 
se référer en ces termes à la musique de danse cadienne dans la réalisation de son documentaire Cajun Country pour la série « American Patchwork» durant les années 1990. Les traductions d'Alan dans le cadre de ce film continuent de révéler ses difficultés en français louisianais. Ainsi commence une chanson de quête ancienne, interprétée par Canray Fontenot :

Fais trois tours de la table ronde.

Fais trois tours de la table ronde.

Allons en chercher, chercher, chercher, chercher.

Les sous-titres de Lomax donnent «Let's sashay, sashay, sashay, sashay» comme équivalent de la dernière ligne, malgré mes propres efforts de l'en convaincre autrement. On me cite néanmoins comme consultant linguistique dans le générique.

Au milieu des années 1930, lorsque John et Alan sont venus au sud de la Louisiane pour la première fois, l'américanisation des Cadiens et des Créoles était très avancée. Les groupes de musique de danse mettant l'accordéon en vedette commençaient à perdre en importance, tandis que des groupes d'instruments à cordes comme les Hackberry Ramblers et les Four Aces de Leo Soileau se sont mis à adopter un style plutôt anglo-américain, en incorporant à leurs répertoires le western swing, le country et les succès radiophoniques. L'arrivée de l'électricité en milieu rural a permis l'amplification sonore dans les salles de danse, ce qui a provoqué l'évolution du style instrumental et du chant. Bien que la nouvelle musique populaire ait en quelque sorte entraîné le refoulement de la musique traditionnelle cadienne et créole, les enregistrements Lomax montrent que cette musique traditionnelle n'était pas disparue.

Les enregistrements commerciaux, centrés sur le mode public de la musique cadienne, ont à peine touché la musique jouée dans les maisons ou " home music », y compris le chant a cappella et la musique instrumentale individuelle. Les Lomax se sont efforcés de couvrir le terrain ignoré par les compagnies commerciales. À la différence de ces compagnies qui sortaient les musiciens de leur contexte culturel pour les enregistrer dans des grands centres comme la Nouvelle-Orléans, Chicago et New-York, les Lomax, eux, pénétraient le milieu social des musiciens et des chanteurs équipés de la machine enregistreuse de la Library of Congress, capable de produire des disques en aluminium sur le terrain.

Il conviendrait peut-être mieux de parler de « déplaçable » plutôt que de «portatif» pour décrire ces premiers enregistreurs. La machine que nous utilisions en Louisiane pesait environ trois cents livres et comprenait un gros amplificateur à tubes, un haut-parleur, un enregistreur de disques et deux énormes 
piles alcalines pour l'électricité. L'enregistreur fonctionnait à l'aide d'un stylet lesté attaché à une vis sans fin, qui gravait la surface d'un disque en aluminium. Si on le compare à la technologie actuelle, le son était plutôt à basse fidélité, avec un bruit de fond, mais dans cent ans, lorsque les enregistrements sur bande magnétique et sur plastique seront tombés en poussière, les originaux des disques cadiens seront bons comme au premier jour, parce que l'aluminium est presque aussi résistant à l'usure du temps que l'or?.

Ils ont enregistré des ballades, des complaintes blues, des chansons à boire et des rondes, ainsi que quelques airs de violon et des groupes de musique de danse. Puisqu'ils ont porté leur attention sur les plus anciennes expressions musicales, les sons qu'ils ont documentés remontent plus loin que les enregistrements commerciaux consacrés aux styles contemporains, et jettent une lumière sur les racines de la musique française en Louisiane.

Les jukeboxes des bars à bière et les émissions des radios locales faisaient tourner en boucle une nouvelle musique qu'on appelait fais-do-do et qui ressemblait beaucoup à la bonne vieille musique émanant de Nashville. Comme cette musique cadienne d'un nouveau genre, faite pour s'amuser, faisait l'objet d'enregistrements et de diffusions commerciales, nous nous sommes concentrés, dans nos enregistrements, sur les tout premiers styles louisianais sans accompagnement, dont nous craignions qu'ils ne soient étouffés par la musique des villes, avec ses orchestrations. L'histoire nous a donné raison, puisque les beaux morceaux que vous entendez dans ces enregistrements, qu'il était très facile de trouver à l'époque, ont désormais quasiment disparu, tandis que l'irrésistible son du fais-do-do et du zydeco prend désormais presque toute la place dans le monde musical des Cadiens... Notre expédition n'a duré que quelques semaines. Imaginez ce que nous aurions trouvé si nous avions pu rester plus longtemps... Cela dit, comme nous n'avions pas beaucoup d'argent ni de temps, je suis content que nous ayons pu préserver ces vieux styles, parce qu'ils donnent un aperçu des racines complexes de la musique cadienne et créole d'aujourd'hui et, avec un peu de chance, ils pourront donner naissance à une musique française en Louisiane qui sera plus riche que le zydeco et le fais-do-do d'aujourd'hui ${ }^{8}$.

Comme résultat de ses premières expériences de terrain en Louisiane, Alan Lomax a commencé à s'intéresser vivement au sort de la région. Comme il a plus tard expliqué « J'y ai bu mon premier verre de vin, mangé mes premières crevettes à la créole, connu ma première véritable aventure amoureuse et fait mes premiers enregistrements indépendants sur le terrain. ${ }^{9} \gg$ La question de

7. Alan Lomax, LCCM.

8. Alan Lomax, LCCM.

9. Miles Hawthorne, «Alan Lomax : Folklorist Seeks "Cultural Equity" », The Daily Advertiser, Lafayette, 20 juin 1980. 
l'avenir de la culture cadienne et créole s'est greffée à sa politique d'égalité culturelle. En tant que membre du Newport Folk Festival Board, il a œuvré pour que l'on envoie Ralph Rinzler au sud de la Louisiane en 1964. Rinzler a contribué puissamment à la cause de préservation culturelle en Louisiane française ; il a aidé Dewey Balfa à développer son talent de porte-parole culturel en assurant sa participation à de nombreux festivals, aux enquêtes sur le terrain et en organisant le premier Hommage à la musique acadienne en 1964. En 1980, on invitait Alan à prononcer le discours d'ouverture au congrès annuel de la Louisiana Folklore Society. Il en a profité pour mettre en cause le legs du Council for the Development of French in Louisiana (CodofIL) et suggérer que cet organisme aurait eu un impact tant négatif que positif, n'ayant pas voulu considérer certains aspects culturels et sociaux dans ses efforts de préservation et de renouveau de la langue française en Louisiane, surtout dans la salle de classe. Selon Woody Baird de l'Associated Press :

«J'ai été horrifié de découvrir que le programme était mis en œuvre par des personnes qui ne parlaient pas cadien », déclare [Lomax]. «C'est comme si on faisait enseigner ici l'anglais en tant que langue seconde par un diplômé d'Oxford. »

Lomax, associé de recherche de l'Université Columbia à New-York, indique que les effets d'un tel programme sont subtils, mais dangereux. « Vous avez un véritable mélange de styles folkloriques qui pourrait être perturbé et déformé de façon irréversible si on utilisait le mauvais style de langue parlée dans les écoles », explique-t-il. «Le style culturel est l'une des principales valeurs créées par l'homme et les Cadiens ont créé leur propre style, qui a survécu pendant toutes ces années. Mais, avec les meilleures intentions du monde, le système scolaire de Louisiane est en train d'attaquer ces valeurs. » [...] Les critiques comme Lomax disent que CoDofiL pourrait faire autant de tort qu'il a fait de bien si l'organisme ne surmonte pas son préjugé négatif à l'encontre du français cadien, par opposition au français standard. ${ }^{10}$

Dans un reportage sur le même discours d'ouverture, Miles Hawthorne, rédacteur du Daily Advertiser de Lafayette, s'est inspiré de la terminologie lomaxienne pour écrire sa manchette : «Folklorist Seeks "Cultural Equity” ». Il a souligné d'ailleurs que :

Les efforts visant à enseigner le français européen aux enfants cadiens entraîneront une dilution de la culture cadienne. «Domengeaux a essayé de mettre la Louisiane dans la poche des critiques et de la culture de la France », explique Lomax. Il dit que de tels efforts sont une force de « centralisation

10. Woody Baird, «Expert Wants Cajun Taught by Natives », dépêche télégraphique, Associated Press, 20 juin 1980. 
stupide » et qu'ils trouvent leur racine dans la cour de Louis XIv, qui rêvait d'une "petite France dans le golfe du Mexique ». Il ajoute : "Le rêve a disparu. $»^{11}$

En conclusion, il souligne le défi lancé par Lomax :

« Il faut que nous battions Louis XIV », déclare Lomax, qui ajoute avec un sourire : « Il faut savoir résister à ces petits tyrans qui sont à la botte de Louis XIV. $»^{12}$

En prononçant ces mots, Lomax avait certainement réussi à susciter la controverse. Le président du CoDofiL, James Domengeaux, était toujours ébranlé par l'affaire Faulk, un enseignant de français au niveau secondaire qui avait tenté d'insérer du matériel pédagogique en français cadien dans les salles de classe de la région et, ce faisant, avait révélé le penchant du CoDOFIL pour le français standard. Cela malgré le fait que le CoDofIL avait parrainé les sept premiers Festivals de musique acadienne ${ }^{13}$. Domengeaux était furieux lors de cette deuxième attaque par ce qu'il jugeait un agitateur de New-York, et aurait même téléphoné à l'Université Columbia dans le but de faire congédier Lomax. Cependant, on a fini par légitimer les revendications de considération culturelle et d'égalité linguistique dans le cadre de l'éducation française en Louisiane, car le Département d'éducation de l'État procédait à l'embauche de Richard Guidry et d'autres enseignants louisianais qui ont mené la lutte pour la « louisianification » de l'enseignement du français dans la région.

Alan était un chercheur exigeant et prolifique. J'ai eu l'occasion de l'observer et d'apprécier ses méthodes en personne lors de son séjour en Louisiane pendant les années 1980 pour la réalisation du volet cadien et créole de la série documentaire «American Patchwork». Sa perspective sur certaines choses n'avait guère changé en toutes ces années. Une fois, il m'avait demandé si je ne connaîtrais pas un jeune gars qu'il pourrait embaucher pour quelques sous et qui lui servirait de chauffeur pour ses excursions sur le terrain. Il est clair qu'il s'intéressait, entre autres, à retracer ses pas. À un moment donné, nous avions entrepris plusieurs voyages à Jennings et Lake Arthur en vue de retrouver certains des hommes qui avaient chanté des jurés pour lui en 1934. Les chanteurs principaux, Oscar Coleman et Jimmy Peters, étaient tous les deux décédés, mais Lomax avait réussi à trouver un des chanteurs secondaires, un certain M. Brown. Rendus chez lui, Lomax s'était présenté à $\mathrm{M}$. Brown, et lui avait demandé s'il se rappelait de lui. M. Brown

11. Miles Hawthorne, op. cit., 20 juin 1980.

12. Loc. cit.

13. Barry-Jean Ancelet, « A Perspective on Teaching the "Problem Language" in Louisiana », The French Review, vol. 61, nº 3, 1988, p. 345-356. 
s'était alors exclamé : «Bien sûr ! Cela fait des années que je dis aux gens que j'ai enregistré un disque, et personne ne m'a jamais cru. Entrez immédiatement et dites à ma famille ce que j'ai fait. » Lomax était entré pour confirmer son histoire et avait fait du vieillard un héros.

Alan se révélait parfois très intimidant. Dewey Balfa nous avait toujours invité à la prudence dans nos relations avec Alan car, selon Dewey, il exerçait un pouvoir et une influence considérables dans le monde du folklore. Il opérait à une échelle démesurée. Aussi, il s'intéressait toujours à la vue d'ensemble. Cependant, toutes les vues d'ensemble sont inévitablement composées d'une multitude de données infimes. Bien qu'on ait critiqué ses théories de « cantométrie » et de « choréométrie », elles ont toutefois l'intérêt d'expliquer certains aspects de l'évolution culturelle et des fusions interculturelles. Parfois ses interprétations de données pouvaient déboucher sur de curieux résultats. Il envisageait en grande partie la culture cadienne et créole en termes de machisme et de répression sexuelle et sociale des femmes. Dans le documentaire "American Patchwork», le portrait qu'il a tracé de Dennis McGee et de sa femme, de toute évidence frustrée, a suscité la gêne chez de nombreuses personnes. Son affirmation dans ce même film que l'avenir de la langue française en Louisiane repose sur le peuple houma a également fait lever plus d'un sourcil. Surtout son interprétation du Mardi Gras y était semblable à celle qu'il avait évoquée au cours d'un atelier de la Smithsonian Institution en 1983, où Dewey Balfa (nous participions à la même table ronde animée par Charlie Camp) m'avait dit à l'oreille : « Ce n'est pas juste. Il faut que quelqu'un s'exprime pour corriger cela. » Je lui avais répondu : « Si tu as quelque chose à dire, dis-le. » Et lui de me rétorquer : « Non. C'est pour cela qu'on t'a envoyé à l'école. » J'avais alors proposé, aussi poliment que possible, une autre interprétation de la structure sociale du Mardi Gras fondée sur mes années de recherche au sein de la communauté. Je venais de terminer mon intervention lorsque Charlie a annoncé la fin de la table ronde, nous remerciant de notre participation. Dans les coulisses, Lomax m'avait admonesté : «N'essaye plus jamais de me contredire en public, espèce de jeune fils de garce. » J'avais riposté en des termes qui me venaient d'une enfance passée dans le nord de la ville de Lafayette : «Écoute, Alan, tu n'es plus dans les jupes de ta mère. Si tu as quelque chose à dire, c'est ton affaire de le dire. J'avais quelque chose à dire et je l'ai dit. » Nous nous sommes réconciliés éventuellement pour collaborer à ce projet «American Patchwork». Ce genre de relation conflictuelle tombait tout à fait dans les habitudes de Lomax. J'étais frustré de constater qu'il avait retenu son interprétation du Mardi Gras dans le film, malgré nos efforts de donner une autre perspective sur cette question et sur d'autres, y compris l'association 
entre le zydeco et les Pygmées, l'association entre les Acadiens et les Huguenots, et aussi la répression sexuelle qui s'exprimerait par les voix aiguës et étranglées qui caractérisent la musique cadienne.

Sur le terrain, il exigeait toujours le meilleur de tout le monde, y compris lui-même. Il surmenait régulièrement les caméramen, les preneurs de son et les consultants. Il demandait aux musiciens et aux chanteurs de refaire leurs chansons devant la caméra, toujours à la recherche de l'exécution parfaite. Il réussissait souvent à enregistrer des performances qui frôlaient la perfection, mais finissait aussi par aliéner les musiciens, du moins temporairement. Un soir, j'avais dû apaiser Canray Fontenot après que Lomax lui eut extirpé une interprétation sublime de sa composition « Barres de la prison », l'ayant intimé de la rejouer maintes fois déjà. Du point de vue d'Alan, Canray était un génie et un géant musical qui venait de se dépasser en offrant une performance hors pair.

Et pourtant, malgré quelques problèmes, il est incontestable que l'héritage Lomax a grandement contribué à assurer l'avenir de la culture cadienne et créole en Louisiane. On entend l'influence des enregistrements Lomax dans la musique contemporaine de groupes tels que Veillée, Feufollet, Beausoleil, les Magnolia Sisters et les Mamou Playboys. D'ailleurs, les enregistrements Lomax ont permis l'interprétation des thèmes poétiques de la musique cadienne et des origines stylistiques de la musique zydeco.

J'ai pris connaissance de la collection Lomax auprès de Ralph Rinzler qui m'a appris que les enregistrements louisianais se trouvaient à la Library of Congress. Je me suis informé quant à leur emplacement et j'ai fini par obtenir une copie de tout le matériel ayant rapport à la Louisiane française pour le nouveau Cajun and Creole Folklore Center à l'University of Southwestern Louisiana (l'actuelle University of Louisiana at Lafayette). Lorsque je me suis assis dans les archives avec le violoneux cadien Michael Doucet pour écouter les enregistrements de terrain pour la première fois, nous étions tous les deux étonnés devant l'abondance d'information qui allait nous obliger à remettre en question l'histoire et le développement de la musique cadienne et créole. Les enregistrements de Lomax des performances de juré chez les Créoles noirs près de Jennings représentaient, pour nous, le premier grand bouleversement. Lorsque je les ai entendus, je me suis immédiatement rendu compte qu'il s'agissait là des origines du zydeco. Certaines des chansons comportaient même la célèbre phrase « Les haricots sont pas salés ». Il est clair que les musiciens créoles noirs, surtout Amédé Ardoin, enregistrés par les compagnies de disques commerciales pendant les années 1930, faisaient aussi partie de l'histoire du zydeco, mais il n'y avait aucun doute que ces jurés constituaient le chaînon manquant. 
Le fait de travailler avec deux chanteurs spirituels révérés m'avait suffisamment familiarisé avec la communauté de Jennings, alors un soir pendant la semaine des jeunes m'ont amené à une église baptiste du coin et m'ont montré, avec leurs amis, le style de danses chantées qu'ils interprétaient couramment lors des activités sociales à l'église. Pendant que j'installais mon micro et que je tirais le câble pour me rendre à ma Model $\mathrm{A}$, dans laquelle se trouvait mon enregistreuse, certains des garçons et des filles se sont mis en couple et ont commencé à danser dans l'église, dans une sorte de danse glissée, secouée, tout à fait semblable aux cris des Sea Islands que nous avons tous appris à connaître. La musique émanait d'un trio de chanteurs et de percussionnistes. Le meneur improvisait de nouveaux couplets et le duo accompagnateur répondait en y superposant un refrain répété. Tous trois produisaient en même temps, en frappant des mains et des pieds, un accompagnement polyrythmé de feu, qui, de loin, donnait l'impression qu'on avait un ensemble de percussionnistes africains dans l'église. Deux des chansons, "Rockaway » et " Feel Like Dying In His Army », avaient des paroles religieuses qui étaient clairement appropriées dans le contexte. Les chansons « J'ai fait le tour du pays », «S'en aller chez Moreau » et « Je veux me marier », chantées en français créole, étaient à caractère profane et sont probablement des incarnations pleinement développées du style juré dont on sait maintenant qu'il était très répandu dans les communautés créoles noires de l'ouest de la Louisiane. $^{14}$

L'histoire des enregistrements des jurés est encore plus impressionnante que la description qu'en fait Alan. Il m'a dit qu'il avait enregistré ces performances de cris et de rondes dans la communauté créole noire en Louisiane malgré les autorités régionales qui soupçonnaient l'intérêt qu'il portait à cette communauté. Il racontait aussi qu'on l'avait emmené en ville en cachette sur la banquette arrière d'une voiture pour enregistrer une performance en soirée. Celle-ci se serait terminée par une bagarre, dont il aurait enregistré les débuts.

Alan a ultimement profité de la parution d'enregistrements provenant de la Louisiane française sur un double album lancé par Swallow Records pour ajouter sa propre perspective à la trentaine de pages d'annotations. Il a saisi l'occasion d'appliquer ses intérêts ethnomusicologiques à sa propre collection. Par rapport aux chanteurs de ballades cadiennes, Lennis Vincent et Sidney Richard, de Kaplan, il précise :

Le duo masculin qui interprète les grandes mélodies symétriques et diatoniques de «J'ai marié un ouvrier» (III : 7), « Madame Gallien » (III : 8) et « Je m'ai fait une maîtresse » (III : 9) chante d'une manière qui convient tout à fait à ces splendides chansons de mariage. L'interprétation est uniforme, la coordination rythmique est plutôt bonne, en dépit de la fréquence des ritardandos rythmiques qui permettent à la mélodie de suivre les couplets de style plutôt fleuri, et le duo

14. Alan Lomax, LCCM. 
parvient à un niveau modeste d'harmonie tonale, en dépit de ses qualités vocales plutôt rugueuses et masculines. ${ }^{15}$

À partir de cette description, il serait difficile de deviner les circonstances de cette session d'enregistrement. Plusieurs années plus tard, Lennis Vincent a expliqué que lui et son cousin Sidney avaient rencontré Lomax à Kaplan un dimanche matin. Ils avaient emprunté le cheval de leur grand-père pour aller à l'église, mais ils avaient fini par abandonner la voie du salut et avaient abouti dans un bar. Ils avaient entendu Lomax parler de son projet. Quelqu'un dans le bar avait suggéré qu'il les enregistre. Ils avaient accepté et étaient sortis à l'extérieur avec Lomax à la recherche d'un coin tranquille. Finalement, ils s'étaient retrouvés dans la goulotte d'une rizerie de la région où ils avaient enregistré ces "grandes mélodies symétriques diatoniques ». Pendant les années 1980 lorsque je leur avais apporté des copies de ces enregistrements, Vincent et Richard ont expliqué qu'ils n'avaient jamais pu en parler à cause des circonstances. En fait, Richard s'était seulement souvenu des évènements après qu'on l'ait considérablement encouragé en ce sens. Lorsqu'il avait entendu sa propre voix dans les enregistrements, il s'était exclamé : « Mais... on dirait que c'est moi! »

Lorsque j'ai offert des copies des enregistrements que John et Alan avaient réalisés de la famille Hoffpauir aux membres de la famille à New-Iberia, un autre aspect de la valeur de ces enregistrements m'a frappé. Tim Hoffpauir avait organisé une réunion de famille pour recevoir les copies. En écoutant, en compagnie de la famille, Elita Hoffpauir chanter une belle ballade française qu'elle avait apprise de son père Julian, j'avais remarqué une femme qui pleurait doucement à l'arrière de la salle. « C'est un moment lourd d'émotion, n'est-ce pas ? », avais-je remarqué. «Oui, » me répondit-elle, "c'est ma mère. Elle est morte quand j'étais jeune. Tout le monde dans la famille me dit qu'elle chantait très bien. C'est la première fois que j'ai l'occasion de l'entendre moi-même. »

Lomax avait également profité du lancement de ces enregistrements provenant de la Louisiane française pour y appliquer ses théories émergeantes de « cantométrie » :

« Je m'endors » (III : 2) est une chanson de blues franco-américaine très originale. Les paroles sont importantes ici et sont prononcées de façon précise, avec une quantité considérable d'embellissements et de trémolos, et le rythme s'adapte aux paroles, dans le style rubato-parlando. Mais les grands intervalles toniques et les notes de blues dans les cadences descendantes donnent à cette vieille mélodie européenne une couleur qui relève plutôt du blues. ${ }^{16}$

15. Alan Lomax, LCCM.

16. Alan Lomax, LCCM. 
Des études antérieures sur la musique cadienne s'étaient consacrées surtout aux éléments stylistiques, ainsi qu'aux questions historiques et socioculturelles relatives à son évolution ${ }^{17}$. À part Louisiana French Folk Songs d'Irene Whitfield Holmes ${ }^{18}$, peu se sont penchés sur le contenu thématique et textuel des paroles des chansons de la Louisiane française, ce qui permettrait des comparaisons entre cette musique et ses traditions parentes en Amérique du Nord française (Québec, Ontario, Acadie des Maritimes, quartier Old Mines du Missouri, la région du vieux Détroit, la région de l'ancien Illinois et les régions franco-américaines du Nord-Est) et d'autres parties du monde francophone (par exemple, la France, les Antilles et l'Océan Indien). Cela s'explique sûrement par plusieurs raisons. Premièrement, en tant que poésie orale, l'on considère que la chanson est plus stable textuellement que le conte et la légende, par exemple, et ainsi plus résistante à l'évolution. Deuxièmement, la langue française des chansons est plutôt impénétrable pour certains des amateurs de musique cadienne d'aujourd'hui. Troisièmement, la musique cadienne contemporaine sert essentiellement à danser et les paroles s'avèrent d'importance secondaire pour beaucoup de participants et d'observateurs chez qui les intérêts sociaux priment. Aussi, certains chercheurs comme Catherine Blanchet ont remarqué qu'il existe une différence essentielle entre la plus ancienne tradition a cappella que l'on appelle «home music» et le son plus contemporain de musique de danse cadienne, accentuée à plus forte raison par le processus de créolisation en Louisiane française. C'est surtout à cause de l'influence des percussions africaines improvisées, qui tient la musique cadienne tellement à l'écart stylistiquement de ses équivalents acadien et québécois, que les comparaisons se révèlent problématiques.

Pourtant, il semblerait peu probable que les musiciens cadiens aient fabriqué de toutes pièces l'ensemble des paroles de musique pour danser qu'ils ont commencé à enregistrer en 1928. Les Lomax ont extensivement documenté la tradition de la ballade a cappella en Louisiane française en 1934, soit six ans après les premiers enregistrements commerciaux de musique de danse cadienne et créole, et de nouveau pendant les années 1960 par l'intermédiaire de Harry Oster et de Ralph Rinzler. J'ai moi-même enregistré un nombre considérable de chansons a cappella à partir des années 1970. Un riche fonds textuel était alors disponible. Selon certains des enregistrements

17. Voir John Broven, South to Louisiana : The Music of the Cajun Bayous, Gretna, Pelican Press, 1983 ; Ann Allen Savoy, Cajun Music. A Reflection of a People, Eunice, Bluebird, 1984 ; Barry-Jean Ancelet, The Maker of Cajun Music, Austin, University of Texas Press, 1984 ; Id., Cajun Music : Origins and Development, Lafayette, University of Southwestern Louisiana Center for Louisiana Studies, 1989.

18. Irene Whitfield Holmes, Louisiana French Folk Songs, Baton Rouge, Louisiana State University Press, 1939. 
commerciaux, il devient évident que le processus d'évolution des ballades vers la musique populaire était en cours dans le contexte de la musique cadienne à l'époque. L'analyse d'autres enregistrements commerciaux des années 1920, 1930, 1940 et même des années 1950 montre qu'il ne s'agissait pas d'un phénomène isolé, bien que l'échelle des transformations dissimule progressivement les assises traditionnelles de la musique populaire. Le même phénomène se produisait également en musique country. La mélodie et les éléments stylistiques se montrent les plus instables dans la plupart des cas. Cependant, ce sont les transformations du contenu poétique des chansons qu'on n'a guère étudiées.

Les chansons et les histoires qui continuent de retentir au sein d'une communauté sont celles qui ont tendance à survivre. Les détails peuvent varier légèrement tandis que le cœur de l'histoire demeure inchangé. Par exemple, les thèmes de la flore et de la faune peuvent changer pour refléter les réalités naturelles. L'évolution de la pratique de la construction peut s'exprimer dans les chansons portant sur les bateaux, les maisons et d'autres constructions. Les références historiques peuvent aussi se transmuter en fonction du contexte. Dans une série de versions de «La Veuve aux deux maris ", un homme part en guerre peu après son mariage. Il promet à sa nouvelle épouse de rentrer bientôt à la maison, mais finit par s'absenter beaucoup plus longtemps qu'il prévoyait. À son retour, il apprend que son épouse, le croyant mort, a l'intention de se remarier le jour même. Elle remarque : "Ce matin j'étais veuve, ce soir je me trouve avec deux maris ». La période d'absence du mari peut varier de 10 à 12 ans dans certaines versions françaises, par rapport à sept ans dans les versions québécoises, et quatre ans dans les versions louisianaises, ce qui symboliserait les Croisades, la guerre de Sept Ans et la guerre de Sécession ou les Guerres mondiales respectivement.

On a enregistré différentes versions de «La Belle qui fait la morte » d'un bout à l'autre du monde francophone. Généralement, la chanson raconte l'histoire d'une jeune fille enlevée du jardin de son père par trois jeunes capitaines. Dans toutes les versions, le plus jeune la prend par la main blanche (symbole de pureté et d'innocence, courant dans les ballades) et la fait monter derrière lui sur son cheval gris (symbole d'une perte d'innocence). Dans la version française, les capitaines emmènent la jeune fille dans un hôtel de Paris où elle doit passer la nuit avec eux ${ }^{19}$. Dans la version louisianaise interprétée par Julian Hoffpauir à New-Iberia et enregistrée par Lomax, elle passe la nuit avec le plus jeune capitaine seulement ${ }^{20}$. Il est plus probable que

19. Marc Robine, Anthologie de la chanson française : la tradition, Paris, Albin Michel, 1994.

20. Alan Lomax, LCCM. 
l'enlèvement soit motivé par l'amour dans la version louisianaise que dans d'autres versions. Néanmoins, dans toutes les versions elle feint la mort afin de sauver son honneur. Lorsque les capitaines la trouvent de toute évidence morte, ils se demandent où ils vont l'enterrer. Dans toutes les versions, ils décident de la ramener au jardin de son père où ils l'ont d'abord trouvée. Dans les versions québécoise et louisianaise, ils vont l'enterrer dans le jardin de son père juste dessous trois fleurs de lys. Elle se réveille plus tard pour consoler son père en deuil, lui expliquant sa tromperie. Le détail concernant les fleurs de lys est particulièrement intéressant. Dans une autre version de la Louisiane française interprétée par la chanteuse créole Alma Barthélémy et enregistrée par Ralph Rinzler pendant les années 1960, ils trouvent la jeune femme parmi les lauriers blancs mais la ramènent près des fleurs de lys pour l'enterrer. Bien que le jardin de son père puisse être un jardin de fleurs, pourquoi la référence spécifique à la fleur de lys dans les versions nordaméricaines, un détail qui se fait remarquer par son absence de la version française ? Et pourquoi trois fleurs de lys ? Historiquement, les trois fleurs de lys sont le symbole du drapeau royaliste français et ce détail est disparu de la version contemporaine de la chanson telle qu'enregistrée dans la République française. Après la guillotine de Louis XVI durant la Révolution française, on a effacé, voire aboli, de la culture populaire toutes les images et les références à la monarchie avilie. Les gens qui allaient devenir les Cadiens et les Québécois ont quitté la France au XVII siècle, longtemps avant la Révolution, mais dans les communautés nord-américaines d'origine française la fleur de lys persiste en tant que symbole non de la monarchie, mais de « francitude ». Elles figurent sur les drapeaux du Québec et de la région de l'Acadiana ainsi que sur les affiches commerciales et gouvernementales. Leur valeur symbolique est également consacrée par cette chanson.

À poursuivre ce raisonnement, si la jeune fille est enterrée sous les trois fleurs de lys, c'est-à-dire sous le drapeau français, elle est sans doute française. Si l'on présume que ses ravisseurs sont aussi ses adversaires, qui sont-ils alors? Il n'est pas impossible qu'on les considère comme étant anglais. Pourquoi donc le symbole a-t-il survécu ? C'est parce qu'il a retenti, parce qu'il évoque une réalité que les chanteurs et leur public auraient compris au moins de façon subliminale, sinon ouvertement. Il est intéressant de noter qu'il n'existe aucune chanson dans le répertoire cadien traditionnel qui touche directement à la question de la déportation des Acadiens en 1755. Cependant, un grand nombre de chansons cadiennes expriment la douleur et la souffrance, le traumatisme de la séparation et de l'aliénation, la désolation des familles déchirées et de l'errance solitaire qui étaient le produit de l'exil. Parallèlement, il n'y a pas de chansons blues traditionnelles qui parlent directement de 
l'esclavage, mais beaucoup du répertoire blues évoque ses malheureux résultats.

L'aspect traditionnel de la musique cadienne et créole aurait exercé une influence conservatrice. Certaines choses peuvent changer, mais raisonnablement et de telle sorte que lesdits changements sont perçus comme étant culturellement appropriés. Comme beaucoup l'ont souligné, la musique cadienne et créole est le résultat d'un processus d'évolution culturelle remarquable. Néanmoins, il est plausible que des données poétiques emmagasinées en mémoire active ont contribué au développement des paroles de la musique de danse. Les textes semblent évoluer en fonction du contexte. La collection Lomax nous a permis d'étudier le cours de cette évolution. Sans elle, on pourrait croire que la musique cadienne a vu le jour au tournant $\mathrm{du} \mathrm{Xx}^{\mathrm{e}}$ siècle dans le sud de la Louisiane. Cependant, à l'aide de la collection Lomax nous constatons que la musique cadienne a subi une transformation importante à cette époque, mais que ses racines thématiques et poétiques remontent à bien plus loin. Sans les enregistrements de la tradition juré, les origines du zydeco resteraient empreintes de mystère.

Le processus de transformation d'anciennes chansons a cappella en musique de danse continue aujourd'hui, mais de façon un peu différente. Autrefois, on condensait de longues ballades en textes relativement brefs de musique de danse, ce qui cristallisait l'intention des chansons en petites parcelles symboliques et impressionnistes. Par exemple, l'on a resserré les paroles de longues chansons narratives qui explicitaient les péripéties de la malmariée française de façon à obtenir le texte plus concis de la chanson « Jeunes Filles de la campagne ». La ballade française traditionnelle « J'ai fait faire un bateau sur mer » raconte l'histoire d'un homme qui demande une jeune fille en mariage. Elle refuse, mais gentiment, lui expliquant qu'elle est trop jeune encore pour considérer le mariage. «La Valse de la belle » de Shirley Bergeron esquisse des propos très semblables, y compris de multiples références à « la belle », mais en beaucoup moins de détails, afin que son texte puisse fonctionner sur une musique de danse. Cependant, beaucoup de groupes sont actuellement en train de ressusciter d'anciennes chansons, conservant les paroles et les transposant pour la musique de danse. Michael Doucet et Beausoleil ont dépoussiéré plusieurs ballades archivées pour ensuite les transformer en morceaux de musique cadienne contemporaine. Par exemple, la chanson «Madame Gallien» de Lanese Vincent et Sidney Richard, enregistrée par les Lomax, est devenue la chanson « Pierrot Grouillet et Mademoiselle Josette ${ }^{21} »$. Depuis lors, d'autres groupes de musique de danse ont eu l'inspiration de réaliser des arrangements de matériel non

21. Beausoleil, Parlez-nous à boire, Arhoolie, 1984. 
accompagné de la collection Lomax. John et Alan Lomax seraient sûrement intéressés par le fait que ces vieilles chansons se sont renouvelées, car leur intention, clairement exprimée, était de préserver le matériel afin qu'il soit mis à la disposition de générations subséquentes. Je ne suis pas certain qu'Alan ait approuvé la transmutation de ces chansons en musique de danse, mais c'est par ce processus qu'elles restent bien vivantes. Par contre, il aurait sans doute approuvé une autre nouvelle mode. Marce Lacouture, les Magnolia Sisters, Steve Riley and the Mamou Playboys et Feufollet se sont mis récemment à intégrer des versions a cappella de ces anciennes chansons à leurs spectacles et à leurs enregistrements. Beausoleil a fait renaître «Belle $e^{22} »$, une chanson non accompagnée que Lomax a recueillie auprès d'un chanteur identifié seulement comme M. Bornu de Kaplan. Steve Riley and the Mamou Playboys ont réalisé une version non accompagnée de «La Chanson des Savoy ${ }^{23}$ ", mettant en vedette une structure harmonique à quatre voix. Les Lomax avaient enregistré les cousins Sonnier et Brasseaux en train de chanter cette même chanson à Erath. Les Magnolia Sisters et le jeune groupe cadien Feufollet ont réalisé des versions a cappella harmoniques de «Sept Ans sur $m e r^{24} \gg$ qu'Elita Hoffpauir avait chantée pour Lomax à New-Iberia. Feufollet a d'ailleurs réalisé un nouvel arrangement de «Les Clefs de la prison » d'Elita Hoffpauir, mettant en vedette un accompagnement instrumental rock ${ }^{25}$. L'arrangement de Wayne Toups de « Trois Jours après ma mort ${ }^{26}$ » tente de conserver la souplesse du style blues de la version originale «Viens donc t'assir sur la croix de ma tombe » d'Edier Segura. Certains musiciens zydeco, tels que Lynn August, ont entendu parler des jurés et se sont mis à étudier cet aspect négligé de leur héritage musical. Lynn August a intégré des versions brèves de « Dégo Zydeco » de Wildfred Charles et «J'ai fait tout le tour du pays » de Jimmy Peters, intitulées simplement «Juré\#1» et «Juré\#2》 sur son album Creole Cruiser ${ }^{27}$. Voilà quelques exemples parmi d'autres du recyclage contemporain des chansons enregistrées par Lomax dans le sud de la Louisiane en 1934.

Finalement, si Alan Lomax avait mal compris certains des phénomènes culturels qu'il observait, il faisait également preuve de grande compréhension et de sympathie à l'endroit du fonctionnement, des interactions et de la survie des cultures. Il était parfois difficile sur le terrain et dans le milieu universitaire, mais il ne s'est jamais éloigné du principe d'égalité culturelle auquel il s'était

22. Beausoleil, Hot Chili Mama, Arhoolie, 1987.

23. Steve Riley and the Mamou Playboys : Bon Rêve, Rounder 116-616-084-2, 2003.

24. Magnolia Sisters : Prends courage, Arhoolie 439, 1995 ; La Bande Feufollet : Belle Louisiane, Feufollet, 2001.

25. Loc. cit.

26. Wayne Toups and ZydeCajun : Reflections of the Past, D \& R, 2005.

27. Lynn August : Creole Cruiser, Black Top BT-1074, 1992. 
dévoué avec tant d'acharnement. Lorsqu'il était aux prises avec une question d'égalité, il ne démordait pas. Pendant dix ans, pas une seule fois ne m'a-t-il téléphoné sans me demander si on avait commencé à enseigner le français cadien dans les écoles. Plusieurs penseurs contemporains ont appuyé son point de vue sur l'importance de la diversité culturelle et linguistique, notamment le physicien Murray Gell-Mann, récipiendaire du prix Nobel, qui a terminé son dernier livre, The Quark and the Jaguar, en soulignant l'importance de l'ADN culturelle (" cultural DNA ») dans son exposé de cette même problématique ${ }^{28}$. Ses appels à la prudence relativement à l' " universal popular culture ${ }^{29} »$ se rapprochent beaucoup de la mise en garde d'Alan dans son « Appeal for Cultural Equity » contre 1' « uniformisation culturelle » qui serait à surveiller, faute de quoi il n'y aurait bientôt «plus aucun endroit qu'il vaille la peine de visiter ou dans lequel il vaille la peine de séjourner ${ }^{30} »$. Comparons Gell-Mann :

De même qu'il est fou de dilapider en quelques décennies une bonne partie de l'abondante diversité biologique qui est le fruit de milliards d'années d'évolution, il est tout aussi fou de laisser disparaître une telle diversité culturelle humaine, qui est, de façon assez analogue, le fruit de dizaines de milliers d'années d'évolution...

L'érosion des particularités culturelles locales dans le monde n'est pas cependant la conséquence, ni dans son intégralité ni même dans sa majeure partie, du contact avec l'effet universalisant des « lumières » scientifiques. La culture populaire est, dans la plupart des cas, bien plus efficace pour ce qui est d'effacer les distinctions entre deux lieux ou deux sociétés. Cela fait des années que les jeans, la restauration rapide, la musique rock et les séries télévisées américaines envahissent le monde. ${ }^{31}$

et Lomax :

[...] nous nous sommes tellement habitués au spectacle pitoyable des carcasses de cultures mortes ou moribondes dans le paysage humain que nous avons appris à considérer cette pollution de l'environnement humain comme étant quelque chose d'inévitable et même de raisonnable, parce que nous supposons, à tort, que ce sont les musiques et les cultures faibles ou inadaptées que ce processus élimine...

Une telle doctrine va non seulement à l'encontre de l'humanité ; elle est aussi une très mauvaise théorie scientifique. Il s'agit d'un faux darwinisme

28. Murray Gell-Mann, The Quark and the Jaguar : Adventures in the Simple and the Complex, New-York, W. H. Freeman, 1994, p. 338-343.

29. Ibid., p. 342.

30. Alan Lomax, "Appeal for Cultural Equity », Journal of Communications, vol. 27, 1977, p. $125-138$.

31. Murray Gell-Mann, op. cit., p. 338-343. 
appliqué à la culture et en particulier à ses systèmes expressifs, comme le langage musical et l'art. Les études scientifiques des cultures et notamment de leurs langues et de leurs musiques montrent qu'elles sont toutes aussi expressives et aussi adaptées à la communication les unes que les autres, même si elles symbolisent parfois des technologies de niveaux différents [...].

Avec la disparition de chacun de ces systèmes, l'espèce humaine perd non seulement une façon de voir, de penser et de ressentir les choses, mais aussi une manière de s'adapter à telle ou telle région de la planète, pour s'y intégrer et la rendre vivable ; il s'agit de surcroît de l'élimination d'un système d'interaction, d'imagination et de symbolisation dont la race humaine pourrait très bien avoir grandement besoin à l'avenir. La seule manière de faire cesser cette dégradation de la culture de l'homme est de s'engager en faveur des principes de la justice politique, sociale et économique. ${ }^{32}$

Les enregistrements audio et vidéo qu'Alan Lomax a amassés sur plus d'un demi-siècle constituent un véritable trésor qu'il désirait ardemment mettre à la disposition des cultures auprès desquelles il avait réalisé ses entretiens. Il s'est réjoui d'apprendre, il y a plusieurs années, que le meilleur de sa collection de la Louisiane française allait paraître en un double album Swallow (plus tard réédité et développé par Rounder). Il avait généreusement fourni un texte détaillé pour les notes en annexe. Il était encore plus ravi d'entendre que ces enregistrements étaient en train d'influencer la génération montante, contribuant à ce que Dewey Balfa appelait « la vie même de la culture ».

La collection qu'Alan Lomax nous a léguée, aussi importante soit-elle, n'est pas sa seule contribution. C'est lui qui a insisté pour que le Newport Folk Foundation envoie des chercheurs sur le terrain en Louisiane. Ralph Rinzler et Mike Seeger se sont rendus sur les lieux et Dewey Balfa était parmi les musiciens cadiens qui ont participé au festival en 1964. Notre propre Festival de musique acadienne à Lafayette est le résultat direct de l'ovation debout obtenue par Dewey Balfa à Newport. Ce dernier, ainsi que Ralph Rinzler et Mike Seeger, étaient ce que Charles Seeger appelait «cultural guided missiles », influencés directement par Alan Lomax.

Il s'intéressait toujours à la vue d'ensemble. Il proposait volontiers une théorie ou une autre pour tenter d'expliquer le cours des choses. Même lorsqu'il avait visiblement tort, il inspirait ceux qui étaient frustrés par ses conjectures opiniâtres à découvrir les faits de l'histoire afin de réfuter ses affirmations.

Dans sa récente lettre au New York Times en réponse à la notice nécrologique de Jon Pareles « Man of His Time, Voices for All Time », Nick Spitzer affirmait que Lomax n'était « certainement pas un isolationniste » et

32. Alan Lomax, « Appeal for Cultural Equity », op. cit. 
qu' « il voulait que les interprètes traditionnels soient en mesure de s'adapter aux réalités économiques et politiques de la société moderne ${ }^{33} \gg$. Je suis d'accord avec lui ; Lomax considérait très sérieusement l'égalité culturelle et la responsabilité du folkloriste envers les gens ordinaires.

Nous sommes ainsi devenus les champions des gens ordinaires du monde, qui ne bénéficient pas de l'appui de la presse, des radios ou de flottes de B-29. Nous croyons à la tradition orale, nous croyons à la situation des petites cultures, nous pensons que certains de ces gens du monde ont quelque chose de valable sur le plan culturel, moral, etc.

Je propose maintenant que nous servions de ponts et que nous établissions un système de communication dans les deux sens. Nous, qui nous exprimons au nom des gens du marché d'ici, avons des obligations vis-à-vis des personnes que nous représentons. ${ }^{34}$

En Louisiane, il a essayé d'amener et d'encourager les Cadiens et les Créoles à lutter pour un avenir où la musique cadienne et ce qu'on appellerait plus tard le zydeco sauraient survivre et faire concurrence aux forces qui autrefois menaçaient leur existence. La grande popularité des musiciens cadiens et créoles aujourd'hui laisse entendre que ces efforts auraient réussi. Que cela produise des effets contradictoires est une autre paire de manches, mais il est indéniable que les musiques cadienne et zydeco font preuve d'une grande vitalité, tout comme les penseurs issus de ces mêmes cultures. En travaillant avec Lomax nous en avons appris long au sujet de l'interprétation de phénomènes culturels, de l'argumentation et de l'intégration de traditions à la culture populaire. Ceux et celles d'entre nous qui nous réjouissons d'entendre de la musique cadienne à Carnegie Hall et du zydeco à la Library of Congress lui devons une fière chandelle. La générosité de la famille Lomax envers la Louisiane française est une tradition qui se poursuit. La fille de Lomax, Anna Chairetakis, a offert récemment de donner tous les enregistrements provenant de la Louisiane dans le cadre du Patchwork Project à l'University of Louisiana at Lafayette. Le fait de rapatrier ces matériaux précieux et de les mettre au service des cultures qu'ils représentent témoigne d'une très bienveillante réciprocité intellectuelle. Certains d'entre nous avons parfois eu à endurer Alan de son vivant, tout en reconnaissant que sa contribution à la compréhension de nos propres communautés perdure. Il était impossible de passer du temps avec lui sans s'ouvrir à de nouvelles perspectives sur le sens et la valeur de la culture traditionnelle. Quelquefois ses méthodes étaient autoritaires, mais plus souvent elles favorisaient des

33. Nicholas R. Spitzer, « Response to Pareles », New York Times, 11 août 2002.

34. Alan Lomax, Selected Writings, 1934-1997. Ed. Ronald D. Cohen, New-York, Routledge, 2003, p. 115-116. 
discussions approfondies sur le sens des choses. Lorsqu'on danse collé-serré, on peut parfois marcher sur les pieds de son partenaire, mais au moins on est en train de danser.

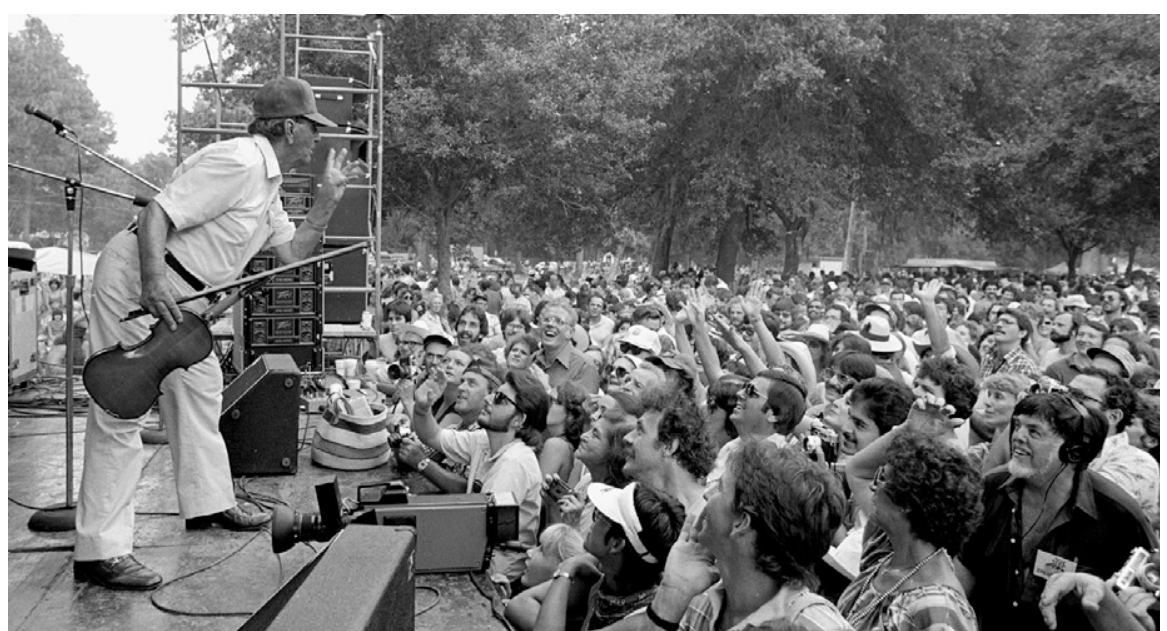

Dennis Mc Gee s'adressant à la foule aux Festivals acadiens de 1982 à Lafayette. Alan Lomax porte des écouteurs en bas à droite. Photographie de Philip Gould, Lafayette.

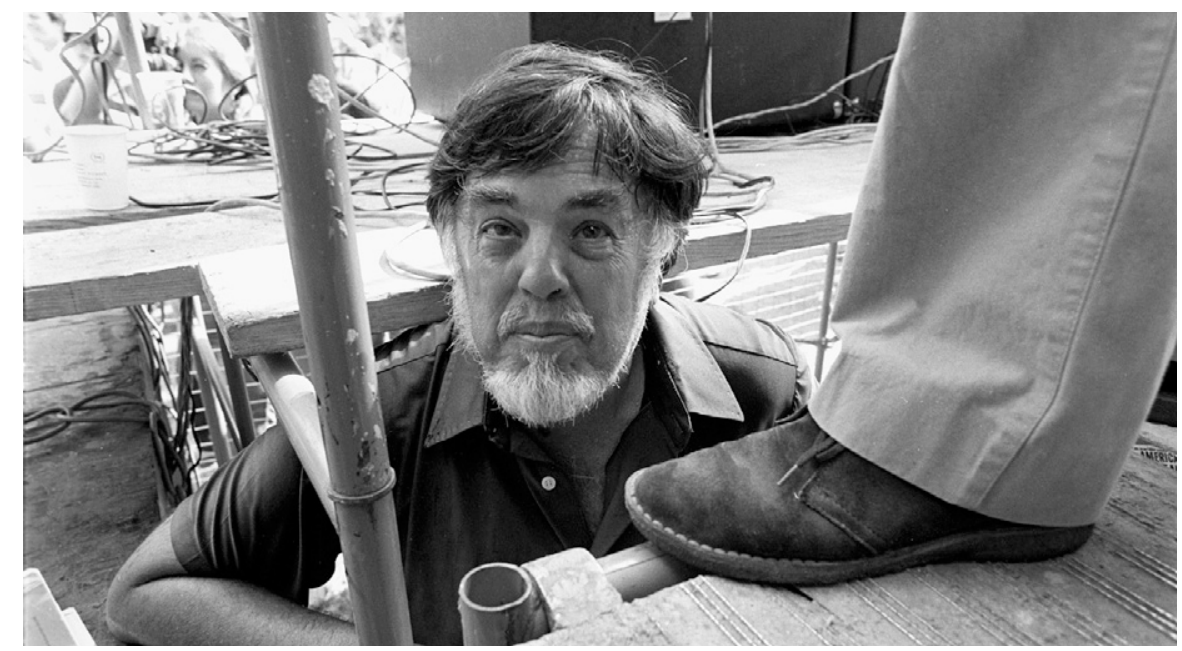

Alan Lomax aux Festivals acadiens de 1982 à Lafayette. Photographie de Philip Gould, Lafayette. 